\title{
Alarmine und ihre Bedeutung für entzündliche Hauterkrankungen
}

\author{
Alarmins and their Role in Inflammatory Skin Diseases
}

Autoren

Institut
Y. Dombrowski, S. Koglin, T. Ruzicka, J. Schauber

Klinik und Poliklinik für Dermatologie und Allergologie, Ludwig-Maximilians-Universität, München
Bibliografie

DOI http://dx.doi.org/

$10.1055 / \mathrm{s}-0030-1255865$

Online-Publikation: 14. 10. 2010

Akt Dermatol 2010; 36:

467-470 @ Georg Thieme

Verlag KG Stuttgart · New York

ISSN 0340-2541

Korrespondenzadresse

Priv.-Doz. Dr. med.

Jürgen Schauber

Klinik und Poliklinik für

Dermatologie und Allergologie

Ludwig-Maximilians-Universität

Frauenlobstr. 9-11

80337 München

juergen.schauber@

med.uni-muenchen.de

\section{Zusammenfassung \\ $\nabla$}

Körpereigene antimikrobielle Peptide (AMP) bilden einen Schutzfilm auf unserer Haut und schützen sie vor bakteriellen und viralen Infektionen. Neben ihrer Funktion in der Infektabwehr initiieren und koordinieren AMPs zudem die kutane Immunantwort und sind als sogenannte „Alarmine“ ein wesentlicher Bestandteil des angeborenen Abwehrsystems. Entzündliche Hautkrankheiten, die mit einer Barrierestörung assoziiert sind, wie das atopische Ekzem, Psoriasis oder Rosazea, sind gekennzeichnet durch eine Fehlregulation des AMP Cathelicidin. Während bei der Rosazea eine fehlerhafte Proteinspaltung von Cathelicidin vor-

\section{Das angeborene Immunsystem der Haut $\nabla$}

Unsere Haut ist die erste Barriere gegen schädliche äußere Einflüsse wie UV-Strahlung, Toxine oder Mikroorganismen. Neben der physischen Barrierefunktion bildet die Haut durch die Sekretion von antimikrobiellen Peptiden eine chemische Schutzschicht aus. Antimikrobielle Peptide (AMP) werden von verschiedenen Zelltypen in der Haut gebildet, wie beispielsweise von Keratinozyten und Sebozyten. Im Rahmen einer Entzündungsreaktion tragen auch die in die Haut infiltrierenden Immunzellen als AMP-Produzenten zur antimikrobiellen Barriere bei [1,2].

Die Bezeichnung „antimikrobielle Peptide“ bezieht sich auf die antimikrobielle Wirkung dieser Peptide gegen ein breites Spektrum an Mikroorganismen, welches über grampositive und -negative Bakterien und Pilze bis hin zu Viren reicht. Neben dieser klassischen Funktion als endogene Antibiotika besitzen viele AMPs auch die Fähigkeit immunregulatorisch zu wirken, indem sie Entzündungsreaktionen initiieren und koordinieren. Diese sogenannte „Alarmin“-Funktion um- liegt, wird beim atopischen Ekzem eine verminderte Produktion diskutiert. Bei Psoriasis wiederum ist Cathelicidin erhöht und möglicherweise an einer Autoimmunreaktion beteiligt. Durch Eingriffe in die Expression von Cathelicidin und anderer AMP bietet sich vielleicht ein neuartiger therapeutischer Ansatz dieser Erkrankungen. Die Cathelicidinproduktion wird in der Haut über den Vitamin D3-Signalweg reguliert, was neue Therapiemöglichkeiten für entzündliche und infektiöse Hautkrankheiten eröffnen könnte. Bis dahin ist die Untersuchung der Regulationsmechanismen der AMPs Gegenstand intensiver dermatologischer Grundlagenforschung.

fasst Effekte auf Komponenten des angeborenen wie auch des adaptiven Immunsystems [3].

Einige AMPs wie Psoriasin, humanes $\beta$-Defensin 3 (HBD3) und RNAse 7 werden von Keratinozyten konstitutiv exprimiert, andere AMP dagegen, wie HBD1 und HBD2 sowie Cathelicidin, werden bei Bedarf gebildet. Ihre Expression wird insbesondere durch mikrobielle Faktoren und inflammatorische Zytokine reguliert [4].

Cathelicidine und $\beta$-Defensine sind die bisher am besten charakterisierten AMP-Familien in der Haut; mehrere Dutzend weitere Proteine mit antimikrobieller Aktivität sind jedoch bekannt [5]. Häufig werden zunächst andere Funktionen der Peptide beschrieben und erst später ihre antimikrobielle Aktivität entdeckt, wie z. B. beim $\alpha$-melanocyte stimulating hormone [6]. Eine Schwierigkeit bei der Identifizierung von AMP ist das Fehlen standardisierter Bioassays zur Charakterisierung ihrer antimikrobiellen Aktivität und die mangelnde Kenntnis ihrer tatsächlich wirksamen In-vivo-Konzentrationen [7]. Der Begriff AMP charakterisiert daher eine heterogene Gruppe von Peptiden mit großer struktureller Variabilität, 


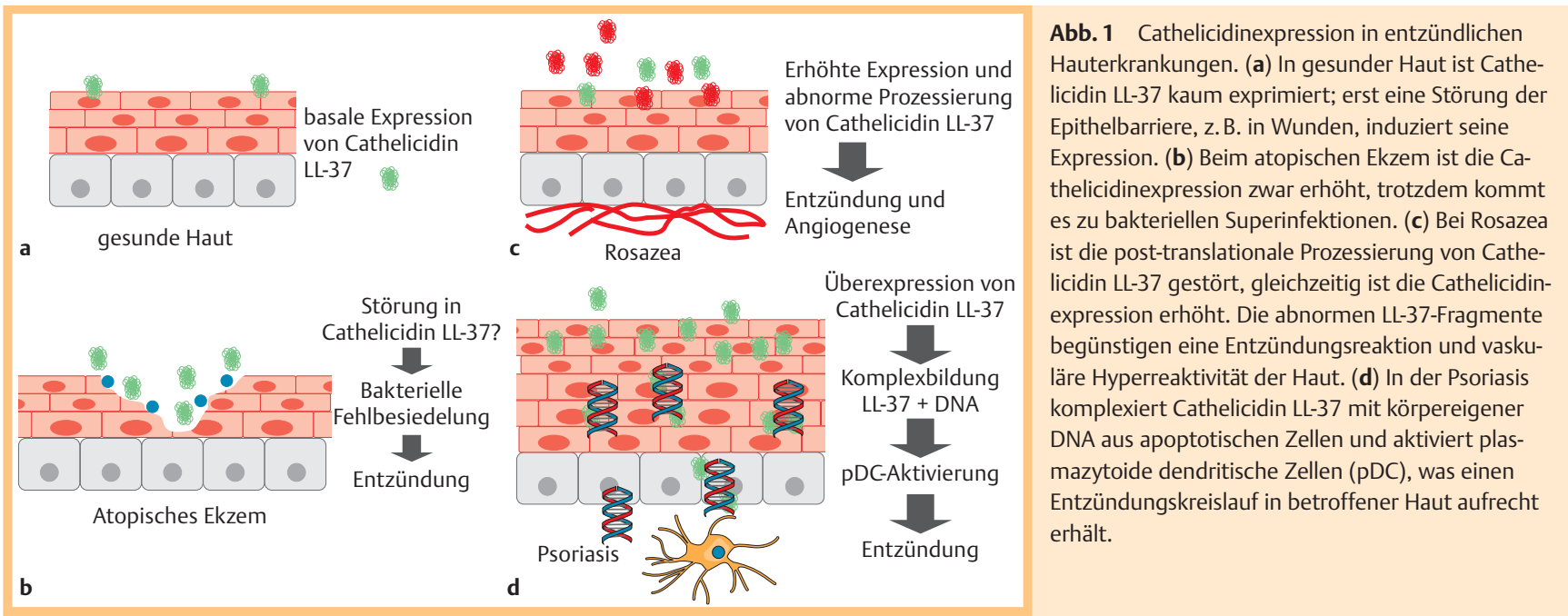

die nur aufgrund ihrer antimikrobiellen Eigenschaften zusammengefasst wird [8].

\section{Cathelicidine: Antimikrobielle Peptide mit multiplen Funktionen \\ $\nabla$}

Die erste in Säugern beschriebene AMP-Familie war die Familie der Cathelicidine. Der Mensch besitzt ein Cathelicidingen (CAMP) [9]. Dieses Gen wird nach Transkription zu einem Vorläuferprotein translatiert und weiter zum aktiven Cathelicidinpeptid LL-37 prozessiert [10]. Das Vorläuferpeptid hCAP18 ist physiologisch inaktiv [11]; erst durch die Prozessierung durch die Serinproteasen Kallikrein 5 und 7 wird das aktive LL-37 generiert [10]. Die antimikrobielle Wirkung von Cathelicidin beruht überwiegend auf der Interaktion mit negativ geladenen mikrobiellen Membranen. Darüber hinaus kann LL-37 bei Säugerzellen rezeptorvermittelte Signalwege aktivieren und dadurch Immunreaktionen initiieren. Beispielsweise beeinflusst LL-37 die Aktivierung von Toll-like-Rezeptoren (TLR) in Immunzellen, die Transaktivierung des „epidermal growth factor“ (EGF)-Rezeptors und die intrazelluläre Kalziummobilisierung [12-14]. Diese über die antimikrobielle Aktivität hinausreichenden Eigenschaften kennzeichnen Cathelicidin als Prototyp-,Alarmin“ in der Infektionsabwehr der Haut.

\section{Die Rolle von Cathelicidin bei entzündlichen Hauterkrankungen}

In gesunder Haut ist Cathelicidin nur gering exprimiert. Hohe Konzentrationen von biologisch aktivem LL-37 finden sich nur bei einer Barrierestörung der Haut, verursacht durch Infektionen, Wunden oder Entzündungen. Bestimmte Hauterkrankungen, wie beispielsweise das atopische Ekzem (AE) oder die Psoriasis, zeichnen sich durch eine Fehlregulation von Cathelicidin aus, was relevant für die Pathogenese dieser Erkrankungen sein könnte.

\section{Atopisches Ekzem}

Bei Patienten mit atopischem Ekzem (AE) kommt es im Rahmen der chronischen Entzündung der Haut zu einer Barrierestörung und häufig zu mikrobieller Fehlbesiedelung. Zunächst wurde angenommen, dass eine verminderte Induzierbarkeit von AMPs wie Cathelicidin oder HBD2 aufgrund des proinflammatorischen Zytokinmilieus in der Epidermis bakterielle oder virale Superinfektionen begünstigt $[15,16]$. Dieses Modell scheint aber simplifiziert, da neuere Studien eine erhöhte AMP-Expression in läsionaler $\mathrm{AE}$ im Vergleich zu gesunder Haut bzw. im Vergleich zu nichtläsionaler AE-Haut belegen [17-19]. Andererseits zeigt eine weitere neue Untersuchung, dass in akuten, oberflächlichen Hautwunden von AE-Patienten AMPs weniger stark induziert werden als in Wunden von gesunden Probanden [20] ( Abb. 1 b). Diese teils widersprüchlichen Ergebnisse legen den Schluss nahe, dass die genaue pathogenetische Rolle der AMPs bei AE noch weiterer intensiver Untersuchungen bedarf.

\section{Rosazea}

Ein fehlreguliertes AMP-Milieu spielt in der Pathogenese der Rosazea eine Rolle, die durch eine Entzündung und vaskuläre Hyperreaktivität der Haut charakterisiert ist. Cathelicidin LL-37 ist in der betroffenen Haut bei Rosazea stark exprimiert und gleichzeitig wird eine abnorme post-translationale Prozessierung von LL-37 zu kleinen Peptidfragmenten beobachtet $(\bullet$ Abb. 1 c). An der Hautoberfläche wird LL-37 von Serinproteasen zu kleinen Peptiden prozessiert, welche verstärkte antimikrobielle Aktivität aber verminderte proinflammatorische Wirkung aufweisen [10,21]. Bei der Rosazea ist die Aktivität der Kallikreine 5 und 7 stark erhöht und es finden sich hier fehlerhaft prozessierte LL-37-Fragmente, die proinflammatorisch und gefäßerweiternd wirken [21].

\section{Psoriasis}

Psoriasis ist eine weitere entzündliche Hauterkrankung, in deren Pathogenese AMPs eine Rolle spielen könnten. Zum einen konnte ein Zusammenhang zwischen einer erhöhten Genkopienzahl von $\beta$-Defensin mit der Inzidenz von Psoriasis hergestellt werden [22]. Zum anderen zeigt sich eine Überexpression von AMPs, insbesondere des antimikrobiellen Peptids Cathelicidin LL-37 in läsionaler psoriatischer Haut, die durch ihre proinflammatorische Aktivität zum Krankheitsverlauf beitragen könnten [23]. Kürzlich wurde gezeigt, dass Cathelicidin LL-37 körpereigene DNA, die bei erhöhtem Zellumsatz im Gewebe extrazellulär vorliegen kann, bindet und komplexiert. DNA-LL-37-Komplexe initiieren dann über die Aktivierung von TLR9 eine Interferon (IFN)-Antwort in plasmazytoiden dendritischen Zellen ( $\mathrm{pDCs}$ ). IFN wiederum aktiviert T-Zellen und führt zu einer nachfolgenden Entzündungsreaktion in der 
betroffenen Haut [24]. Im Rahmen dieser Immunreaktion wird weitere körpereigene DNA aus apoptotischen Zellen freigesetzt, welche wiederum von LL-37 gebunden werden kann. Dieser Kreislauf der DNA-Freisetzung, LL-37-Komplexierung und pDCAktivierung perpetuiert möglicherweise die Entzündungskaskade in läsionaler psoriatischer Haut ( $\bullet$ Abb. 1 d).

Die gestörte Expression und Funktion von AMPs, insbesondere Cathelicidin, in der Pathogenese von infektiösen und chronischentzündlichen Hauterkrankungen eröffnet möglicherweise neue Ansatzpunkte für die Therapie. Strategien zur Wiederherstellung des physiologischen AMP-Milieus in erkrankter Haut setzen jedoch eine genaue Kenntnis der zugrunde liegenden Regulationsmechanismen voraus.

\section{Regulation der Cathelicidinexpression über den Vitamin D-Signalweg \\ $\nabla$}

Bis zur Identifizierung einer Vitamin D-Rezeptorbindungsstelle im Promotor des Cathelicidingens waren die molekularen Mechanismen der Cathelicidinexpression unbekannt [25]. Inzwischen belegen zahlreiche Studien, dass Cathelicidin direkt durch Vitamin D3 reguliert wird [26-28]. Auch weitere Cathelicidin-Regulationsfaktoren wie Co-Aktivatoren und epigenetische Veränderungen sind inzwischen identifiziert [29].

In Keratinozyten wird die Vitamin D3-Vorläuferform 7-Dehydrocholesterol unter UV-Einfluss zu inaktivem Vitamin D3 (Calciol) umgesetzt. In der Leber erfolgt die erste Hydroxylierung von Calciol zu Calcidiol durch die Vitamin D-25-Hydroxylase (CYP2R1). In der Niere folgt die zweite Hydroxylierung von Calcidiol zu aktivem Vitamin D3 (Cacitriol) durch CYP27B1. Keratinozyten können Calciol unabhängig von Leber und Niere hydroxylieren. Dies ist für das kutane Mikromilieu relevant, da Keratinozyten aktives Vitamin D3 zur Proliferation und Differenzierung sowie zur Aufrechterhaltung einer intakten antimikrobiellen Barriere benötigen.

\section{Mögliche therapeutische Beeinflussung von Cathelicidin bei entzündlichen Hauterkrankungen \\ $\nabla$}

Durch Beeinflussung des Vitamin D-Metabolismus und damit Cathelicidin, beispielsweise durch UV-Exposition, könnte sich die Möglichkeit bieten, die Infektionsabwehr der Haut zu beeinflussen und Einfluss auf chronisch entzündliche Hautkrankheiten mit Beteiligung von Cathelicidin zu nehmen [30].

\section{Atopisches Ekzem (AE)}

In der Therapie des AE wird häufig eine UVB-Fototherapie eingesetzt. Der Erfolg dieser Behandlung könnte auf die UVB-abhängige Induktion von $\beta$-Defensinen [31] und eine Vitamin D3-Aktivierung und nachfolgende Cathelicidininduktion in der betroffenen Haut zurückzuführen sein [32,33] und nicht allein, wie bisher angenommen, auf den Einfluss auf T-Zell-mediierten Immunantworten. Neben der UVB-Strahlentherapie könnte auch eine orale Vitamin D3-Gabe oder eine topische Applikation positive Effekte zeigen. Da die topische Applikation von Vitamin D3 bzw. seiner Analoga in Tierversuchen Hautirritationen und Ekzeme auslösen kann, erscheint eine orale Gabe günstiger. Tatsächlich sind kleinere Pilotstudien, bei denen eine orale Gabe von Vitamin D3 bei AE gegeben wurde, inzwischen publiziert worden [34]. Größere Studien insbesondere mit Untersuchung klinischer
Endpunkte (u.a. mikrobielle Besiedelung, Rezidivfreiheit) fehlen allerdings noch.

\section{Rosazea}

Bei Rosazea liegt eine gesteigerte Cathelicidinexpression und fehlerhafte Proteinprozessierung in den betroffenen Hautarealen vor. Ziel einer möglichen Therapie könnte sein, das Cathelicidinprofil bei Rosazea zu normalisieren. In Einzelfallberichten sind Vitamin D-Rezeptor-Polymorphismen mit der Pathogenese der Rosazea assoziiert [35]. Durch Eingriff in den Vitamin D-Signalweg ließe sich die Cathelicidinexpression blockieren, was zu einer Verbesserung des Krankheitsbildes führen kann. Ein möglicher weiterer therapeutischer Ansatzpunkt könnte in der Blockade der Proteaseaktivität in der Haut bei Rosazea liegen. Die Prozessierung von Cathelicidin erfolgt durch Serinproteasen, welche durch $\alpha 2$-Makroglobulin oder andere Serpine inaktiviert werden könnten [36]. Interessanterweise hemmen Tetracycline, die bereits erfolgreich in der Therapie der Rosazea eingesetzt werden, kutane Proteasen [37].

\section{Psoriasis}

Wie bei Rosazea liegt auch bei der Psoriasis eine erhöhte Cathelicidinexpression vor. Strategien, die den Kreislauf aus Nukleinsäure-LL-37-Komplexierung, pDC- (und mDC-)Aktivierung und Inflammation unterbrechen, wären als antientzündliche Strategien denkbar [24,38]. Paradoxerweise verbessert die Applikation von Vitamin D3-Analoga die Entzündungsreaktion bei Psoriasis, obwohl dies zu einem gesteigerten LL-37-Spiegel führt und damit die Entzündung verschlimmern sollte [39]. Eine molekulare Erklärung dieses Paradoxons steht bislang aus. Möglicherweise bewirken Vitamin D3-Analoga eine alternative Prozessierung und Aktivierung von Cathelicidin LL-37 oder aber die Wirkung geht über die des Vitamin D-Signalweges hinaus. Die genauere Untersuchung der Expression von Cathelicidin wird auch ein besseres Verständnis der Wirkweise von Vitamin D3-Analoga in der Therapie der Psoriasis ermöglichen.

\section{Schlussfolgerung \\ $\checkmark$}

Alarmine wie Cathelicidin bieten sich als mögliche Zielstrukturen für neue Therapien entzündlicher Hauterkrankungen mit gestörter AMP-Expression an. Bevor allerdings Therapieempfehlungen formuliert werden können, müssen die zugrunde liegenden molekularen Mechanismen genauer analysiert werden.

\section{Abstract}

\section{Alarmins and their Role in Inflammatory Skin Diseases $\nabla$}

Our skin is constantly exposed to potentially harmful microorganisms. As effectors of innate immunity antimicrobial peptides (AMP) protect the skin from bacterial or viral infections. Furthermore, these peptides are an essential part of the host's immune system as they initiate and coordinate immune reactions. Hence, the term „alarmins“ has been coined to designate AMPs. Inflammatory skin diseases such as atopic eczema, rosacea and psoriasis are characterized by dysfunction or dysregulation of antimicrobial peptides. In rosacea, cathelicidin peptide processing is abnormal, whereas in atopic eczema the induction of cathelicidin may be diminished. In psoriasis cathelicidin expression is upregulated 
and may be involved in an autoimmune cascade. Pharmacological regulation of AMP expression could be a novel therapeutic strategy to ameliorate inflammation and hence the burden of these inflammatory diseases. Therefore, an in-depth understanding of molecular mechanisms underlying AMP regulation is needed and current subject of dermatological research.

\section{Literatur}

1 Braff MH, Zaiou M, Fierer J et al. Keratinocyte production of cathelicidin provides direct activity against bacterial skin pathogens. Infect Immun 2005 Oct; 73 (10): $6771-6781$

2 Agerberth B, Charo J, Werr J et al. The human antimicrobial and chemotactic peptides LL-37 and alpha-defensins are expressed by specific lymphocyte and monocyte populations. Blood 2000 Nov; 196 (9): $3086-3093$

3 Schauber J, Gallo RL. Expanding the roles of antimicrobial peptides in skin: alarming and arming keratinocytes. J Invest Dermatol $2007 \mathrm{Mar}$; 127 (3): $510-512$

4 Harder J, Glaser R, Schroder JM. Human antimicrobial proteins effectors of innate immunity. J Endotoxin Res 2007; 13 (6): 317- 338

5 Braff MH, Gallo RL. Antimicrobial peptides: an essential component of the skin defensive barrier. Curr Top Microbiol Immunol 2006; 306: $91-110$

6 Cutuli M, Cristiani S, Lipton JM, Catania A. Antimicrobial effects of alpha-MSH peptides. J Leukoc Biol 2000 Feb; 67 (2): 233-239

7 Schauber J, Gallo RL. Antimicrobial peptides and the skin immune defense system. J Allergy Clin Immunol 2008 Aug; 122 (2): 261 - 266

8 Peric M, Koglin S, Ruzicka T, Schauber J. [Cathelicidins: multifunctional defense molecules of the skin]. Dtsch Med Wochenschr 2009 Jan; 134 $(1-2): 35-38$

9 LarrickJW, Lee J, Ma S et al. Structural, functional analysis and localization of the human CAP18 gene. FEBS Lett 1996 Nov 25; 398 (1): 74-80

10 Yamasaki K, Schauber J, Coda A et al. Kallikrein-mediated proteolysis regulates the antimicrobial effects of cathelicidins in skin. FASEB J 2006 Oct; 20 (12): $2068-2080$

11 Zaiou $M$, Nizet $V$, Gallo RL. Antimicrobial and protease inhibitory functions of the human cathelicidin (hCAP18/LL-37) prosequence. J Invest Dermatol 2003; 120 (5): 810-816

12 Tokumaru S, Sayama K, Shirakata $Y$ et al. Induction of keratinocyte migration via transactivation of the epidermal growth factor receptor by the antimicrobial peptide LL-37. J Immunol 2005 Oct 1; 175 (7): $4662-4668$

13 Di Nardo A, Braff MH, Taylor KR et al. Cathelicidin antimicrobial peptides block dendritic cell TLR4 activation and allergic contact sensitization. J Immunol 2007 Feb 1; 178 (3): 1829-1834

14 Tomasinsig L, Pizzirani C, Skerlavaj B et al. The human cathelicidin LL37 modulates the activities of the P2X7 receptor in a structure-dependent manner. J Biol Chem 2008 Nov 7; 283 (45): 30471 - 30481

15 Ong PY, Ohtake T, Brandt C et al. Endogenous antimicrobial peptides and skin infections in atopic dermatitis. N Engl J Med 2002 Oct 10; 347 (15): $1151-1160$

16 Nomura I, Goleva E, Howell MD et al. Cytokine milieu of atopic dermatitis, as compared to psoriasis, skin prevents induction of innate immune response genes. J Immunol 2003; 171 (6): 3262 - 3269

17 Ballardini N, Johansson C, Lilja G et al. Enhanced expression of the antimicrobial peptide LL-37 in lesional skin of adults with atopic eczema. Br J Dermatol 2009 Jul; 161 (1): 40 - 47

18 Glaser $R$, Meyer-Hoffert $U$, Harder J et al. The antimicrobial protein psoriasin (S100A7) is upregulated in atopic dermatitis and after experimental skin barrier disruption. J Invest Dermatol 2009 Mar; 129 (3): $641-649$
19 Harder J, Dressel S, Wittersheim $M$ et al. Enhanced expression and secretion of antimicrobial peptides in atopic dermatitis and after superficial skin injury. J Invest Dermatol 2010 May; 130 (5): 1355 - 1364

20 Mallbris L, Carlen L, Wei T et al. Injury downregulates the expression of the human cathelicidin protein hCAP18/LL-37 in atopic dermatitis. Exp Dermatol 2010 May; 19 (5): $442-449$

21 Yamasaki K, Di Nardo A, Bardan A et al. Increased serine protease activity and cathelicidin promotes skin inflammation in rosacea. Nat Med 2007 Aug; 13 (8): 975 - 980

22 Hollox EJ, Huffmeier U, Zeeuwen PL et al. Psoriasis is associated with increased beta-defensin genomic copy number. Nat Genet 2008 Jan; 40 (1): $23-25$

23 Henseler T, Christophers E. Disease concomitance in psoriasis. J Am Acad Dermatol 1995 Jun; 32 (6): $982-986$

24 Lande R, Gregorio J, Facchinetti $V$ et al. Plasmacytoid dendritic cells sense self-DNA coupled with antimicrobial peptide. Nature 2007 Oct 4; 449 (7162): $564-569$

25 Wang T-T, Nestel F, Bourdeau Vet al. Cutting Edge: 1,25-Dihydroxyvitamin D3 is a direct inducer of antimicrobial peptide gene expression. J Immunol 2004; 173: 2909-2912

26 Gombart AF, Saito T, Koeffler HP. Exaptation of an ancient Alu short interspersed element provides a highly conserved vitamin D-mediated innate immune response in humans and primates. BMC Genomics 2009; 10: 321

27 Weber G, Heilborn JD, Chamorro Jimenez CI et al. Vitamin D induces the antimicrobial protein hCAP18 in human skin. J Invest Dermatol 2005 May; 124 (5): 1080 - 1082

28 Gombart AF, Borregaard N, Koeffler HP. Human cathelicidin antimicrobial peptide (CAMP) gene is a direct target of the vitamin $\mathrm{D}$ receptor and is strongly up-regulated in myeloid cells by 1,25-dihydroxyvitamin D3. FASEB J 2005 Jul; 19 (9): 1067 - 1077

29 Schauber J, Oda Y, Buchau AS et al. Histone acetylation in keratinocytes enables control of the expression of cathelicidin and CD14 by 1,25-Dihydroxyvitamin D(3). J Invest Dermatol 2008 April; 128 (4): 816-824

30 Dombrowski Y, Peric M, Koglin S et al. Control of cutaneous antimicrobial peptides by vitamin D3. Arch Dermatol Res 2010 Aug; 302 (6): $401-408$

31 Glaser R, Navid F, Schuller W et al. UV-B radiation induces the expression of antimicrobial peptides in human keratinocytes in vitro and in vivo. J Allergy Clin Immunol 2009 May; 123 (5): 1117-1123

32 Vahavihu K, Ala-Houhala M, Peric $M$ et al. Narrowband ultraviolet B treatment improves vitamin $\mathrm{D}$ balance and alters antimicrobial peptide expression in skin lesions of psoriasis and atopic dermatitis. $\mathrm{Br} J$ Dermatol 2010 Aug; 163 (2): 321-328

33 Peric M, Lehmann B, Vashina $G$ et al. UV-B-triggered induction of vitamin D3 metabolism differentially affects antimicrobial peptide expression in keratinocytes. J Allergy Clin Immunol 2010 Mar; 125 (3): 746 749

34 Hata TR, Kotol P, Jackson $M$ et al. Administration of oral vitamin D induces cathelicidin production in atopic individuals. J Allergy Clin Immunol 2008 Oct; 122 (4): 829-831

35 Jansen T, Krug S, Kind P et al. BsmI polymorphism of the vitamin D receptor gene in patients with the fulminant course of rosacea conglobata (rosacea fulminans). J Dermatol 2004 Mar; 31 (3): 244-246

36 Rubin H. Serine protease inhibitors (SERPINS): where mechanism meets medicine. Nat Med 1996 Jun; 2 (6): 632 -633

37 Uitto VJ, Firth JD, Nip L, Golub LM. Doxycycline and chemically modified tetracyclines inhibit gelatinase A (MMP-2) gene expression in human skin keratinocytes. Ann N Y Acad Sci 1994 Sep 6; 732: 140-151

38 Ganguly D, Chamilos G, Lande $R$ et al. Self-RNA-antimicrobial peptide complexes activate human dendritic cells through TLR7 and TLR8. J Exp Med 2009 Aug 31; 206 (9): 1983-1994

39 Peric M, Koglin S, Dombrowski Y et al. Vitamin D analogs differentially control antimicrobial peptide/,alarmin“expression in psoriasis. PLoS One 2009; 4 (7): e6340 